\title{
The Inelastic Scattering of NO by Kr: Rotational Polarization over a Rainbow
}

\author{
Helen Chadwick, ${ }^{\dagger} @$ Bethan Nichols, $^{\dagger}$ Sean D. S. Gordon, ${ }^{\dagger}$ Balazs Hornung, ${ }^{\dagger, \triangle}$ \\ Eleanor Squires, ${ }^{\dagger}$ Mark Brouard, ${ }^{*}, \dagger$ Jacek Kłos, ${ }^{\ddagger}$ Millard H. Alexander, $₫$ F. Javier

$$
\text { Aoiz, }{ }^{\S} \text { and Steven Stolte } \|, \perp, \#
$$

The Department of Chemistry, University of Oxford, The Physical and Theoretical Chemistry Laboratory, South Parks Road, Oxford, OX1 3QZ, United Kingdom, Department of Chemistry and Biochemistry, University of Maryland, College Park, MD, 20742, USA.,

Department of Chemistry and Biochemistry and Institute of Physical Science and Technology, University of Maryland, College Park, MD, 20\%42, USA, Departamento de Química Física, Facultad de Química, Universidad Complutense, 28040 Madrid, Spain., Institute of Atomic and Molecular Physics, Jilin University, Changchun 130012, China., Department of Physics and Astronomy, LaserLaB, Vrije Universiteit, Amsterdam, De Boelelaan 1081, 1081 HV Amsterdam, The Netherlands, and Laboratoire Francis Perrin, Bâtiment 522, DRECEM/SPAM/CEA Saclay, 91191 Gif sur Yvette, France.

E-mail: mark.brouard@chem.ox.ac.uk 


\begin{abstract}
We use molecular beams and ion imaging to determine quantum state resolved angular distributions of NO radicals, after inelastic collision with $\mathrm{Kr}$. We also determine both the sense and the plane of rotation - the rotational orientation and alignment respectively - of the scattered NO. By full selection and then detection of the quantum parity of the NO molecule, our experiment is uniquely sensitive to quantum interference. For forward scattered NO we report hitherto unseen changes in the plane and sense of rotation with scattering angle, and show, remarkably, that the rotation of the NO molecule after collision can be near-maximally oriented for certain transitions and scattering angles. These effects are enhanced by the full parity selection in the experiment, and result from the interplay between attractive and repulsive forces.
\end{abstract}

\title{
Graphical TOC Entry
}

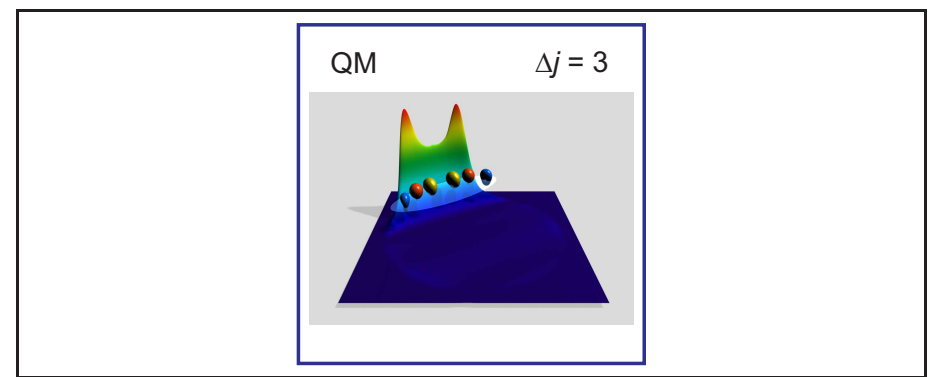

\section{Keywords}

inelastic scattering, angular momentum polarization, " $\ell$-type" rainbow

\footnotetext{
${ }^{*}$ To whom correspondence should be addressed

${ }^{\dagger}$ University of Oxford

†University of Maryland

IUniversity of Maryland

$\S$ Universidad Complutense de Madrid

\| Jilin University

${ }^{\perp}$ Vrije Universiteit

\#DRECEM/SPAM/CEA Saclay

${ }^{\circledR}$ Current address: Laboratoire de Chimie Physique Molculaire, Ecole Polytechnique Fédérale de Lausanne, Lausanne, Switzerland

${ }^{\triangle}$ Current address: School of Chemistry, University of Bristol, Cantock's Close, BS8 1TS, UK
} 
September 2, 2014

Non-equilibrium gases relax most efficiently by transfer of rotational energy. A major objective of the field of molecular collision dynamics is the understanding of how the forces in play affect the distribution of the scattered products in space and among the allowed final rotational states. As this field evolves, we need experiments that can separate the contribution to inelastic scattering of both the long-range (attractive) and short-range (repulsive) regions of intermolecular potentials. Also, a persistent intellectual challenge is uncloaking quantum effects in inelastic molecular collisions.

Collisions of NO with noble gases have long been the paradigm for rotationally inelastic scattering, ${ }^{1}$ and have made possible a fertile give-and-take between increasingly sophisticated experiments and theoretical modeling. Many previous studies have focussed on determining the relative differential cross-section (DCS) of the scattered products, ${ }^{2-7}$ known to be a sensitive probe of the underlying potential energy surface (PES). ${ }^{5,6}$ Despite their sophistication, in most of these experiments the near-degeneracy (called $\Lambda$-doubling) of the selected NO rotational level is not resolved, so that observed transitions are in reality the sum of two processes, in which one conserves and the second reverses the overall parity of the NO rotational wave function. Consequently, the dependence of the efficiency of rotational inelasticity on the quantum parity of the NO levels is obscured.

In our laboratory, we first select and, then, detect a single $\Lambda$-doublet state of the scattered NO. ${ }^{5,6,8}$ We have seen novel structures in the DCSs which depended on the parity of the initial and final $\mathrm{NO}(X)$ rotational levels. In the most sophisticated experiments, ${ }^{8}$ we use polarized lasers to determine the alignment ${ }^{9,10}$ of the rotational angular momentum of the scattered NO. This can provide more details about how the NO molecule is torqued during the collision, ${ }^{3,8,11,12}$ thereby setting a more rigorous challenge to our understanding of the dynamics.

These earlier experiments were done with Ar as a collision partner. ${ }^{8}$ Exploiting the stronger long-range attractive interaction of $\mathrm{NO}$ with $\mathrm{Kr},{ }^{13}$ which has a deeper well $\left(D_{\mathrm{e}} \simeq\right.$ 
$\left.130 \mathrm{~cm}^{-1}\right)$ and a more extended attractive limb compared with $\operatorname{Ar}-\mathrm{NO}(X)^{14}\left(D_{\mathrm{e}}=110 \mathrm{~cm}^{-1}\right)$, we report here a new experimental probe of the role of attractive forces in inelastic scattering, namely the plane and sense of rotation of the NO in the forward scattered region. ${ }^{15}$ Specifically, we detect a pronounced "rainbow" as a function of scattering angle. ${ }^{16-20}$ This type of rainbow phenomenon arises when collision trajectories are deflected by the attractive part of the intermolecular interaction potential and are then amplified by constructive quantum interference. The result is a build-up in scattering intensity at the so-called rainbow angle. The name reflects similarities in the semiclassical description of atomic scattering and the scattering of light by water droplets in the atmosphere. In addition, our new experiments reveal how the interplay between attractive and repulsive forces leads to a remarkable shift with scattering angle of the plane and sense of rotation - the rotational alignment and orientation, respectively $^{9,10}$ - of the inelastically scattered NO in the region of the rainbow.

In the electronic ground state $\left(X^{2} \Pi\right)$ the rotational levels of NO appear in two spin-orbit manifolds, labelled by the magnitude of $\Omega$, the projection of the total electronic angular momentum (exclusive of the nuclear spin) $\boldsymbol{j}$ onto the bond axis. We limit our focus here to rotational transitions within the lower $(\Omega=1 / 2)$ spin-orbit manifold. As observed above, each rotational level is split further into two $\Lambda$-doublet sub-levels. These are the symmetric (labelled $e$, with $\epsilon=+1)$ and antisymmetric $(f, \epsilon=-1)$ combinations of $+\Omega$ and $-\Omega$ wavefunctions. These sub-levels differ in their parity (the symmetry with respect to space fixed inversion), given by $p=\epsilon(-1)^{j-1 / 2}$, and are nearly degenerate, separated by only $\sim 0.01 \mathrm{~cm}^{-1}$ for $j=0.5$.

The unpaired spin and the non-zero electronic orbital angular momentum of NO complicates the scattering. Approach of a spherical collider splits the degenerate $\Pi$ state into two states, of $A^{\prime}$ and $A^{\prime \prime}$ reflection symmetry. The corresponding NO-Kr PESs are degenerate asymptotically but differ in the interaction region. In a semiclassical picture of such a system, collision trajectories can follow one or the other of these two PESs, and can also hop between the two. Just like in the textbook two-slit experiment, quantum interference 
can also occur. In general for such systems the resulting dynamics provides an example of scattering on two coupled PESs. ${ }^{21,22}$

By means of hexapole electric field focussing, we select NO in just the $\Omega=1 / 2, j=0.5, f$ $\Lambda$-doublet level. We cross this fully state-selected NO beam by a beam of $\mathrm{Kr}$ atoms. ${ }^{5,6,8} \mathrm{We}$ use velocity-mapped ${ }^{23}$ ion imaging, ${ }^{24}$ subsequent to $\left(1+1^{\prime}\right)$ resonantly enhanced multiphoton ionization (REMPI), to infer both the rotational quantum number $j^{\prime}$ and the final $\Lambda$-doublet state of the scattered NO. Using a photoelastic modulator, on alternate laser shots we rotate the direction of the electric field of the excitation laser from lying in the plane defined by the molecular beams (horizontal, H) to perpendicular to this plane (vertical, V). This allows us to determine the alignment of $\boldsymbol{j}^{\prime}$. The orientation of $\boldsymbol{j}^{\prime}$ is probed by directing alternately left $(\mathrm{L})$ and right $(\mathrm{R})$ circularly polarized light perpendicular to the plane defined by the molecular beams (see Supporting Information). ${ }^{3}$

Figure 1 presents the summed $\mathrm{H}+\mathrm{V}$ ion images for three spin-orbit conserving, $f \rightarrow f$ transitions, for which the $\Delta j=$ even/odd transitions conserve/reverse the NO parity. ${ }^{21}$ For the low $\Delta j$ transitions considered here, the angular distributions of the scattered $\mathrm{NO}(X)$ are strongly forward peaked. The images show a single peak in intensity for the parityconserving transitions $\left(j^{\prime}=2.5, f\right.$ and $\left.4.5, f\right)$, but three peaks for the parity-changing transition $\left(j^{\prime}=3.5, f\right)$. Fits to the experimental ion images, ${ }^{6,8}$ done as described in the Supporting Information, predict the angular distributions shown in the right panels of Figure 1. Note that the distribution for $\Delta j=3$ shows a strong secondary maximum at $\sim 20^{\circ}$. This peak in the angular distribution is responsible for the extra structure in the corresponding ion image.

The pronounced peak is an " $\ell$-type" rainbow. ${ }^{15,16,19,20,25}$ Here $\ell$ is the magnitude of the orbital (end-over-end) angular momentum of the NO-Kr pair. Low- $\ell$ collisions are headon, while high- $\ell$ collisions are glancing encounters. ${ }^{16,19}$ The classical impact parameter, $b$, is proportional to $\ell$. Because the initial rotational angular momentum of $\mathrm{NO}(X)$ is only $j=0.5, \ell$ is approximately equal to $J$, the total angular momentum of the system. 


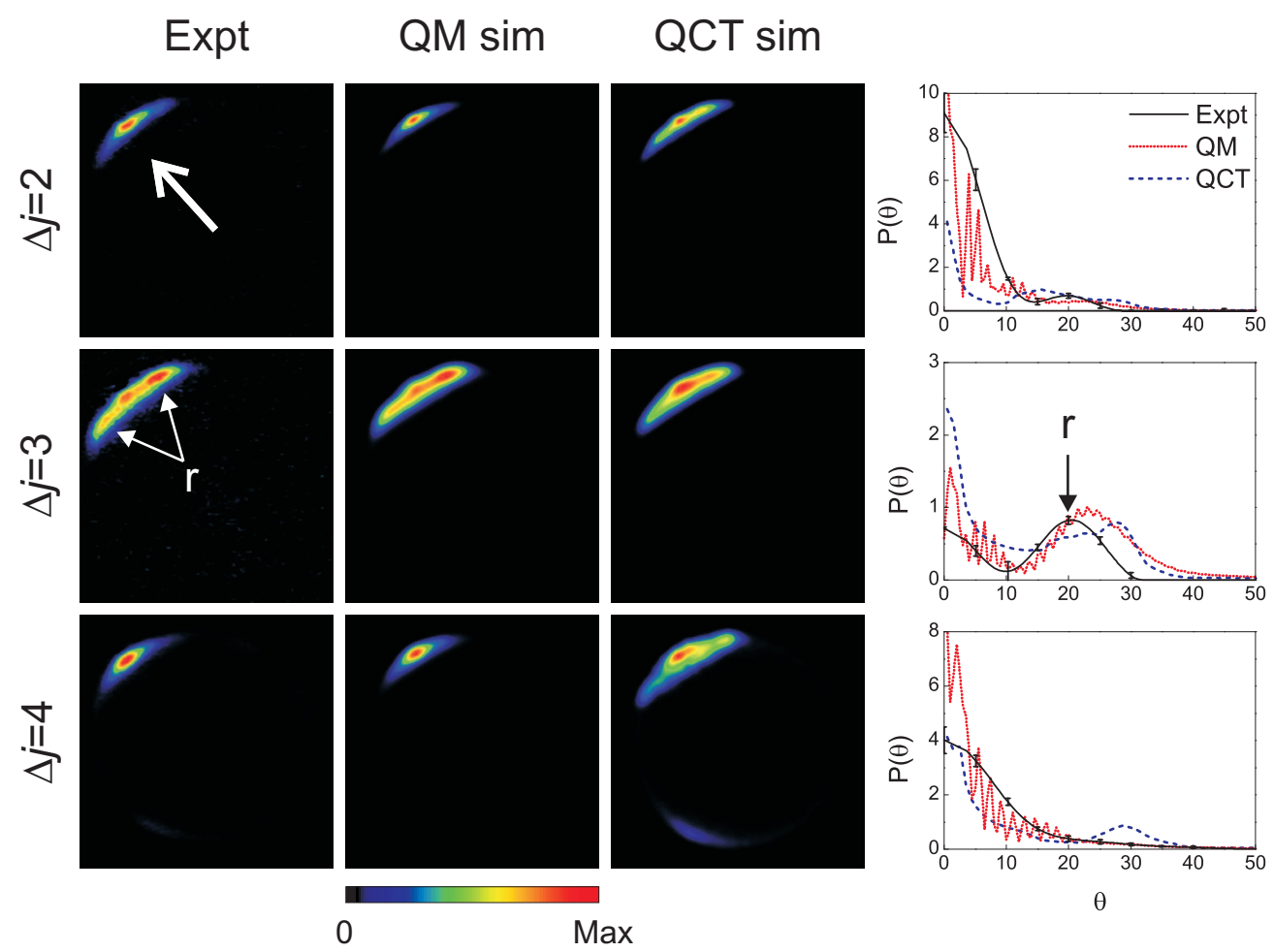

Figure 1: A comparison of the experimental (first column), open-shell QM simulated (second column) and closed-shell quasi-classical trajectory (QCT) simulated (third column) $\mathrm{H}+\mathrm{V}$ ion images for scattering of $\mathrm{NO}(X)$ with krypton from initial state $j=0.5, f$ to final states $j^{\prime}=2.5, f$ (top row), $j^{\prime}=3.5, f$ (middle row) and $j^{\prime}=4.5, f$ (bottom row). The direction of the initial relative velocity is shown as a white arrow in the top left panel. The right column shows the angular distributions of scattered $\mathrm{NO}(X)$ (normalized to unity in the angular range shown) obtained experimentally (black solid line), and from QM (red dotted line) and QCT scattering calculations (blue dashed line) averaged over the experimental collision energy distribution. The position of the rainbow in the ion images and in the angular distributions is labelled with an ' $\mathrm{r}$ ' for $j^{\prime}=3.5, f$ (middle row).

An $\ell$-type rainbow arises from attractive forces between the collision partners, and, exactly as in atom-atom scattering, ${ }^{16,17,20,25}$ can be attributed classically to the focussing of trajectories at scattering angles close to the minimum in the deflection function (the maximum attractive deflection). ${ }^{18}$ This corresponds to the secondary maximum in the classical scattering angle (the absolute value of the deflection function). This is plotted in the panels in the third column of Figure 2. These scattering angles were predicted from quasi-classical trajectory (QCT) calculations, ${ }^{25}$ in which the NO molecule was treated as a closed-shell system, and the trajectories were run on the average of the $A^{\prime}$ and $A^{\prime \prime}$ PESs, designated $V_{\text {sum }}$, 

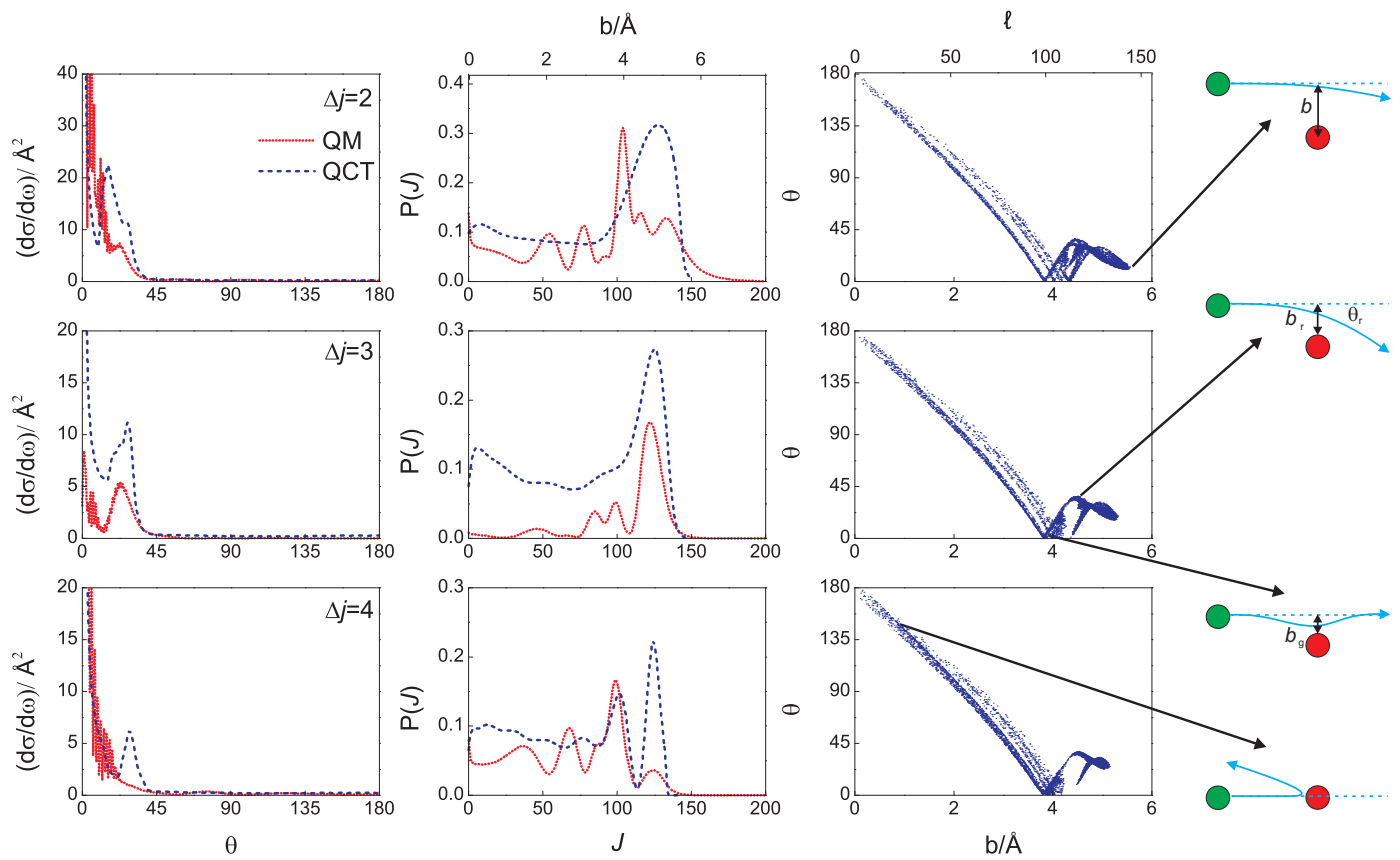

Figure 2: Open-shell QM (dotted red lines) and closed-shell QCT (dashed blue lines) DCSs (first column) and opacity functions (second column), and closed-shell QCT deflection functions (blue dots, third column) for $\Delta j=2$ (top row), $\Delta j=3$ (middle row) and $\Delta j=4$ (bottom row). The QM data are for the $f \rightarrow f \Lambda$-doublet resolved transitions. The DCS and opacity function data have been averaged over the experimental collision energy distribution. The cartoons in the forth column of the figure illustrate classical trajectories at different impact parameters leading into different scattering angle regions, $\theta$. The rainbow angle, $\theta_{\mathrm{r}}$, is illustrated in the second figure down. The third cartoon illustrates scattering at the so-called 'glory' impact parameter, $b_{\mathrm{g}} \sim 4 \AA$ (equivalent to $J \sim 100.5$ at a collision energy of $514 \mathrm{~cm}^{-1}$ ). Glory scattering is mainly responsible for the strong forward scattered peak observed in the $\Delta j=2$ and $\Delta j=4 f \rightarrow f$ transitions, while rainbow scattering is the key feature of the $\Delta j=3 f \rightarrow f$ transition. The fourth (from the top) cartoon illustrates small impact parameter scattering which is dominated by the repulsive part of the potential.

which is largely responsible in this case for spin-orbit conserving transitions. ${ }^{21}$ The scatter in the points in these deflection function plots reflect the (small) variation of the predicted scattering angle with the initial relative orientation of $\mathrm{NO}$ and $\mathrm{Kr}$.

Here, the rainbow arises from scattering at impact parameters ranging from 4 to $5 \AA$. This distance corresponds to the outer attractive wing of the NO-Kr PES. ${ }^{13}$ The plots shown in Figure 2 highlight the classical origin of the peaked structure, and suggest that a rainbow should be observed in the DCSs for all the transitions under consideration. In striking contrast, the rainbow peak is seen experimentally only for the $\Delta j=3, f \rightarrow f$ (parity- 
changing) transition. For the $\Delta j=2$ and $\Delta j=4, f \rightarrow f$ (parity-conserving) transitions the rainbow peaks are masked by the forward scattering process known as glory scattering, ${ }^{16}$ in which the effects of the attractive and repulsive parts of the potential cancel each other resulting in nearly zero deflection angles. This is illustrated by the third cartoon (from the top) on the right side (forth column) of Figure 2. Clearly, to observe these parity-dependent effects necessitates a parity-resolved experiment, as performed here.

We also carried out full quantum (QM) scattering calculations, retaining the open-shell character of the NO molecule and including both the $A^{\prime}$ and $A^{\prime \prime}$ PESs. ${ }^{21}$ We used the PESs of Wen et al. ${ }^{13}$ and the HIBRIDON ${ }^{26}$ suite of codes. From both the QM and QCT DCSs, we simulated the experimental ion images, ${ }^{6,8}$ making use of the methods outlined in the Supporting Information. While classical mechanics can explain qualitatively the origin of the observed rainbow, Figure 1 reveals that the QM images are clearly in better agreement with experiment. The remaining discrepancies between experiment and QM theory, most notably the small difference in the observed $\left(\sim 20^{\circ}\right)$ and QM predicted $\left(\sim 23^{\circ}\right)$ location of the rainbow, reflect the combined uncertainties in the experiments and in PESs employed in the dynamical calculations. Note that the rapid diffraction oscillations ${ }^{7}$ seen in the calculations cannot be observed in the current experiments due to the limitations in angular resolution, and the spread in collision energy. ${ }^{6}$ This is confirmed by the QM simulations of the ion images, which also do not show evidence of rapid diffraction oscillations in the forward direction.

For a given impact parameter, rotational inelasticity can result from trajectories which hit either on the $\mathrm{N}$ side or the $\mathrm{O}$ side of the $\mathrm{NO}$ center of mass. In a quantum treatment, these two trajectories will interfere. For a homonuclear molecule, $\mathrm{N}_{2}$ for example, the interference will be complete, so that collision-induced parity changing collisions will not occur. In a nearly-homonuclear molecule, such as NO, the quantum interference will be substantial, leading, for example, to significant oscillations in the integral cross sections. ${ }^{27}$ 
A QCT simulation treats the two trajectories incoherently, so that similar interference will not occur. ${ }^{6,25,28}$ The QCT simulations predict rainbows for both the parity-conserving and parity-changing transitions, while in the QM simulations, which include quantum interference, the rainbow appears only in the parity-changing transition. In earlier experiments $^{2-4,11,12}$ both parity components of the $j=0.5$ level of NO were present in the initial beam. Here, the hexapole prepares a single NO parity state, which allows a cleaner, more resolved look at the difference between parity-conserving and parity-changing transitions.

Figure 2 (second column) also compares the QCT and QM opacity functions (the probability of inelastic scattering as a function of the impact parameter). We observe that the QCT simulations (a) overestimate the degree of inelasticity and (b) incorrectly weight the glory and rainbow contributions as a function of $b$. Although the QCT method can describe nearly quantitatively many aspects of rotationally-inelastic scattering, our work here reveals its limits - as we might have anticipated - in the prediction of phenomena which are sensitive to quantum interference. We have found a key factor to be the absence of the concept of parity in classical mechanics. As noted above, parity dependent effects have been observed previously for collisions of $\mathrm{NO}+\mathrm{Ar}$, in which the dominant effects can be understood in terms of the different contributions from largely repulsive terms in the PES. ${ }^{6}$ Similar behaviour is also observed for higher $\Delta j$ transitions in $\mathrm{NO}+\mathrm{Kr}$, to be reported elsewhere. ${ }^{29}$ However, the present parity dependent results at low $\Delta j$ are most striking, because they are exquisitely sensitive to the balance of attractive and repulsive terms in the PES.

Significant further insight into the scattering dynamics is provided here by the measurement of the rotational polarization ${ }^{9}$ of the scattered NO in the region of the $\ell$-type rainbow (for further experimental details see the Supporting Information). Rotational alignment information is derived from, specifically, the $(\mathrm{V}-\mathrm{H})$ difference images, shown for example in Figure 3. Classically, the intensity of the absorption leading to ionization is proportional to the square of the cosine between the transition dipole moment, $\boldsymbol{d}$, and the linear electric field of the photon. This is maximized when both are parallel. Thus a positive difference 
image (V more intense than $\mathrm{H}$ ) implies that the transition moment of the scattered NO lies preferentially perpendicular to the scattering plane.

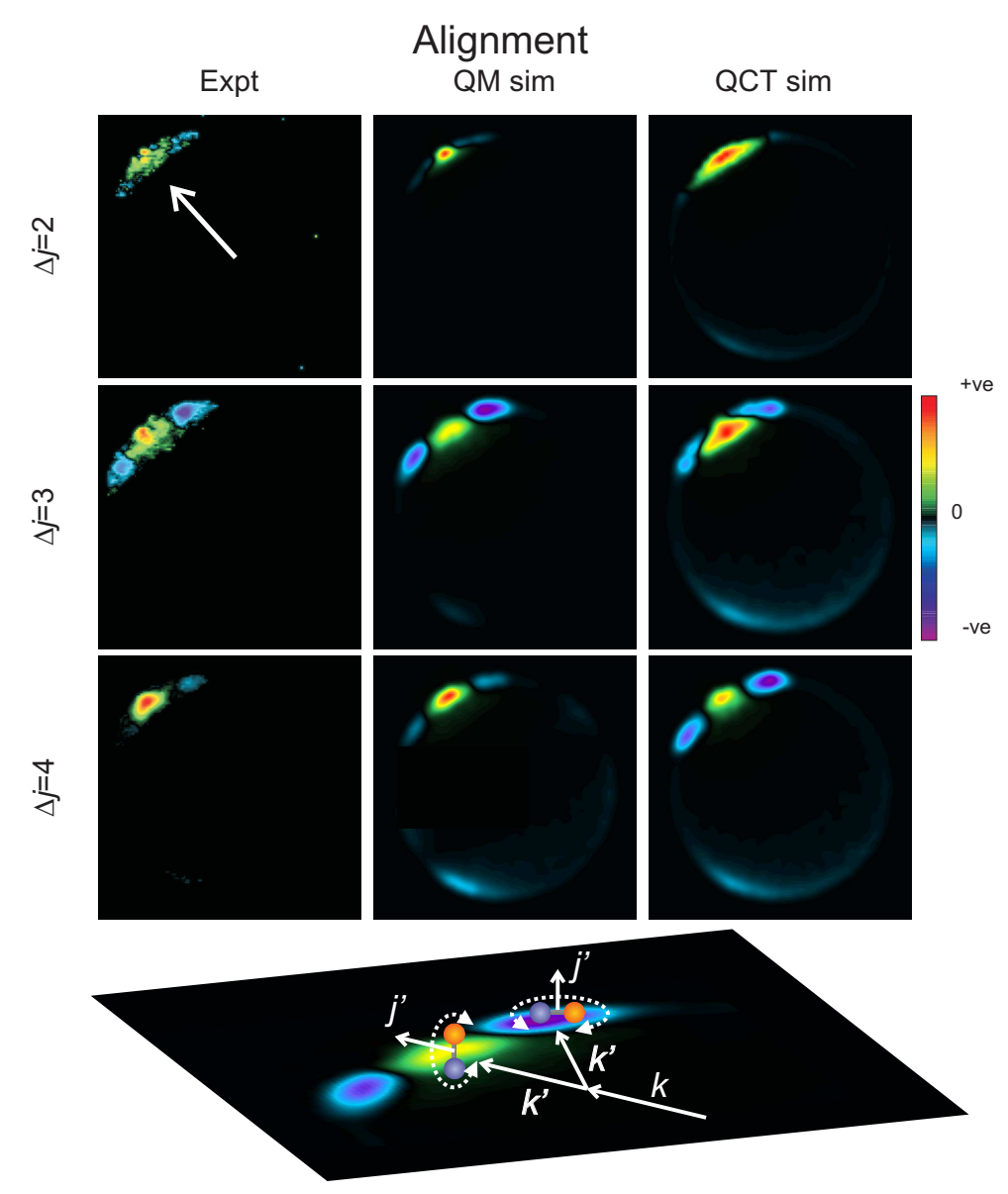

Figure 3: Comparison of the experimental (first column), open-shell QM simulated (second column), and closed-shell QCT simulated (third column) $(\mathrm{V}-\mathrm{H})$ difference alignment ion images for scattering of $\mathrm{NO}(X)$ with krypton from the initial state $j=0.5, f$ to final states $j^{\prime}=2.5, f$ (top row), $j^{\prime}=3.5, f$ (middle row) and $j^{\prime}=4.5, f$ (bottom row). The direction of the relative velocity is shown as a white arrow in the top left panel. The panel at the bottom of the figure illustrates a cartoon of the angular momentum alignment after the collision for $\Delta j=3$.

To record the ion images we used $\mathrm{R}_{21}$ excitation, for which $\boldsymbol{d}$ is perpendicular to $\boldsymbol{j}^{\prime}$ at high $j^{\prime} \cdot{ }^{9}$ Consequently, a positive difference image implies that $\boldsymbol{j}^{\prime}$ lies in the scattering plane. Qualitatively, since $\boldsymbol{j}^{\prime}$ is nearly perpendicular to the molecular axis $\boldsymbol{r}$, this indicates that a positive difference image corresponds to the scattered $\mathrm{NO}(X)$ rotating like a propeller, with motion perpendicular to the scattering plane as depicted in the bottom panel of Figure 3. In contrast, a negative difference image is consistent with $\boldsymbol{d}$ and $\boldsymbol{r}$ lying in, and $\boldsymbol{j}^{\prime}$ perpendicular 
to, the scattering plane. This corresponds to frisbee-like motion.

The experimental difference image goes from positive (red or yellow) to negative (blue) as the scattering angle increases. This corresponds to a slewing of the plane of rotation of the scattered NO rotates from out-of to in the scattering plane. Despite the long interest in polarization in molecular scattering, ${ }^{10,30-32}$ this is the first observation of a pronounced variation in the polarization of an inelastically-scattered molecule in the region of a rainbow. In our case this is particularly apparent because we scatter out of a single parity state. For the parity-conserving transitions $(\Delta j=$ even, $f \rightarrow f)$, the largest difference images, and hence the strongest product rotational alignment, occurs in the forward (glory) scattered region. In contrast, for the parity-changing collisions $(\Delta j=$ odd, $f \rightarrow f)$, the largest difference images, and hence the strongest rotational alignment, occurs in the region of the $\ell$-type rainbow.

We also used the QCT and QM calculations to predict difference images, shown in Figure 3. Once again, it is the QM simulated images that most accurately reproduce experiment. However, compared with the angular distributions shown in Figure 1, the alignment of the final rotational angular momentum is quite well accounted for by the classical simulations (see Supporting Information).

Measurements of the rotational angular momentum orientation of the scattered NO, using the methods detailed in the Supporting Information, serve to reinforce these conclusions. Here we use circularly polarized light to probe the sense of rotation of the NO molecule after the collision. ${ }^{3}$ This information is derived from the $\mathrm{L}-\mathrm{R}$ difference images shown in Figure 4. Negative intensity indicates that right hand circularly polarized light has a higher detection probability than left handed circularly polarized light, which corresponds to a molecule rotating anticlockwise in the plane defined by the molecular beams, as shown in the bottom panel of Figure 4. Likewise, positive intensity indicates clockwise rotation of the NO in the plane of the molecular beams. 


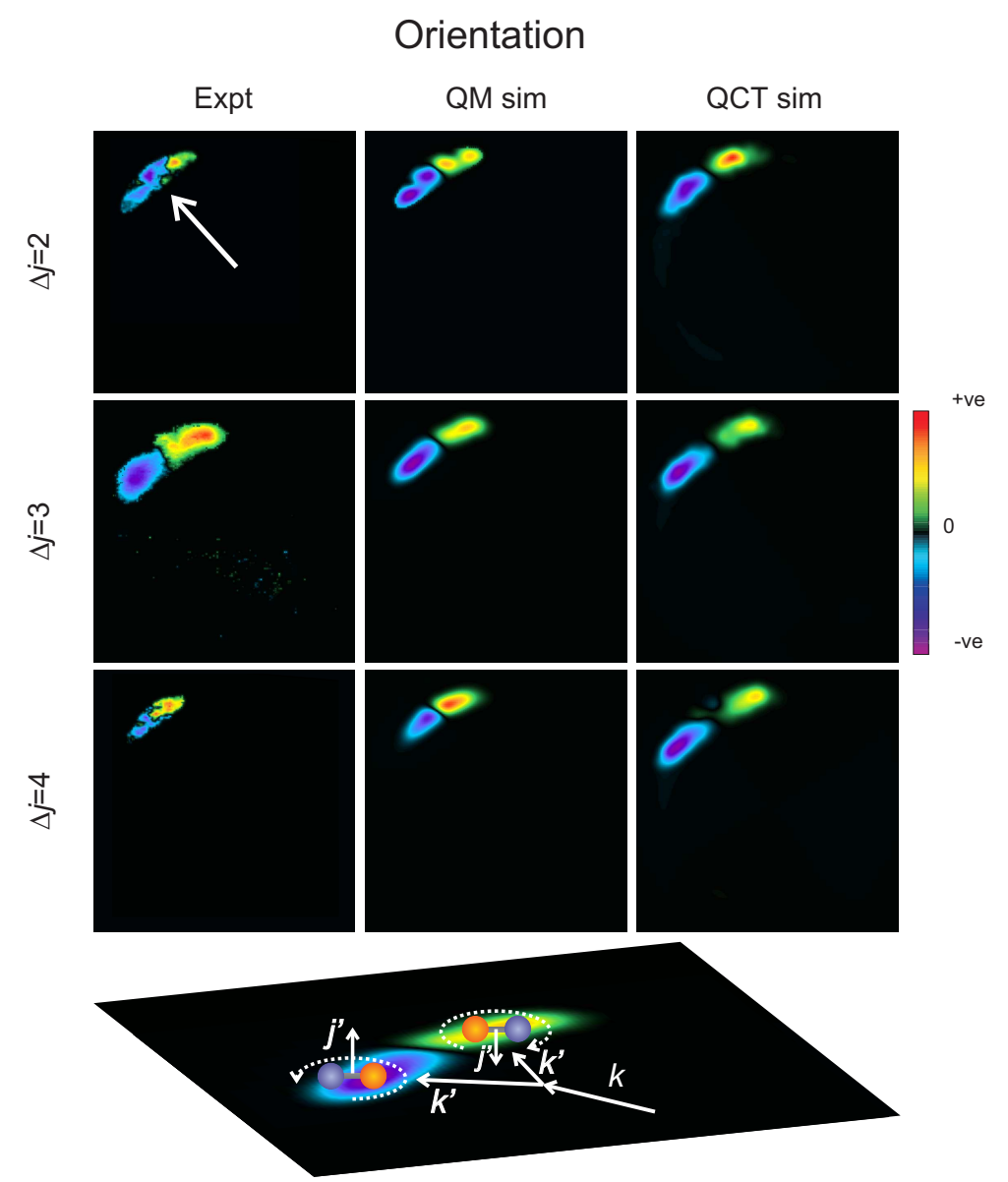

Figure 4: Collision-induced orientation of the NO rotational angular momentum over the rainbow, as reflected in the $(\mathrm{L}-\mathrm{R})$ difference orientation ion images, for scattering of $\mathrm{NO}(X)$ with krypton. The panel at the bottom of the figure illustrates a cartoon of the angular momentum orientation after the collision for $\Delta j=3$.

Although parity conserving and changing transitions show similar orientation behavior, the most intense difference image, and hence the strongest orientation, is seen in the region of the " $\ell$-type" rainbow of the parity changing transition to $j^{\prime}=3.5, f$. In fact, rotation of the scattered NO is near maximally oriented at angles close to the rainbow, corresponding to near complete selectivity in the sense of rotation for this transition (see Supporting Information). In contrast, for parity conserving transitions to $j^{\prime}=2.5, f$ and $j^{\prime}=4.5, f$ the orientation is more pronounced in the forward, glory scattering region. Whilst the QCT data again suggest that the collision-induced orientation of the scattered NO can be understood qualitatively using a classical attractive mechanism, ${ }^{33}$ the QM simulations are required for a quantitative 
modelling of the experiment (see Supporting Information).

To conclude, we have measured the angular distributions and rotational polarization both alignment and orientation - for collisions of fully quantum state resolved NO with Kr. Resolution of the NO initial $\Lambda$-doublet level has allowed observation of an " $\ell$-type" rainbow in the parity changing collision. We show that the rotational angular momentum of the scattered NO in the region of the rainbow can be near-maximally oriented. The sense and plane of rotation of the scattered NO can be understood qualitatively using classical mechanics, and are signatures of the balance between attractive and repulsive forces at play during collision. However, the quantum parity plays a crucial role in influencing the observed angular distributions, and full QM calculations are required for a quantitative description of the experiment.

\section{Associated Content}

\section{Supporting Information}

Supporting Information provides additional details of the experimental, computational and data analysis procedures employed. This material is provided free of charge via the Internet http://pubs.acs.org.

\section{Author Information}

\section{Corresponding author}

*Email: mark.brouard@chem.ox.ac.uk

\section{Notes}

The authors declare no competing financial interest. 


\section{Acknowledgments}

The support of the UK EPSRC (to M.B. via Programme Grants EP/G00224X/1 and EP/ L005913/1), the EU (to M.B. via FP7 EU People ITN project 238671), and the Spanish Ministry of Science and Innovation (grants CTQ2008-02578, CTQ2012-37404, and CSD200900038) are gratefully acknowledged. M.H.A. acknowledges financial support from the US National Science Foundation (grant No. CHE-1213332). S.S. acknowledges support from the National Basic Research Program of China (973 program) under grant No. 2013 CB922200 and from the National Science Foundation of China under grant Nos. 11034003 and 91221301.

\section{References}

(1) Andresen, P.; Joswig, H.; Pauly, H.; Schinke, R. Resolution of interference effects in the rotational excitation of NO $(N=0)$ by Ar. J. Chem. Phys. 1982, rr, 2204.

(2) Suits, A. G.; Bontuyan, L. S.; Houston, P. L.; Whitaker, B. J. Differential cross-sections for state-selected products by direct imaging - Ar + NO. J. Chem. Phys. 1992, 96, 8618.

(3) Lorenz, K. T.; Chandler, D. W.; Barr, J. W.; Chen, W.; Barnes, G. L.; Cline, J. I. Direct Measurement of the Preferred Sense of NO Rotation After Collision with Argon. Science 2001, 293, 2063-2066.

(4) Kohguchi, H.; Suzuki, T.; Alexander, M. H. Fully State-Resolved Differential Cross Section for the Inelastic Scattering of the Open-Shell NO Molecule by Ar. Science 2001, 294, 832-834.

(5) Gijsbertsen, A.; Linnartz, H.; Rus, G.; Wiskerke, A. E.; Stolte, S.; Chandler, D. W.; Kłos, J. Differential cross sections for collisions of hexapole state-selected NO with He. J. Chem. Phys. 2005, 123, 224305. 
(6) Eyles, C. J.; Brouard, M.; Yang, C. H.; Kłos, J.; Aoiz, F. J.; Gijsbertsen, A.; Wiskerke, A. E.; Stolte, S. Interference structures in the differential cross-sections for inelastic scattering of NO by Ar. Nat. Chem. 2011, 3, 597-602.

(7) von Zastrow, A.; Onvlee, J.; Vogels, S. N.; Groenenboom, G. C.; van der Avoird, A.; van de Meerakker, S. Y. T. State-resolved diffraction oscillations imaged for inelastic collisions of NO radicals with He, Ne and Ar. Nat. Chem. 2014, 6, 216-221.

(8) Brouard, M.; Chadwick, H.; Eyles, C. J.; Hornung, B.; Nichols, B.; Aoiz, F. J.; Jambrina, P. G.; Stolte, S. Rotational alignment effects in $\mathrm{NO}(\mathrm{X})+$ Ar inelastic collisions: An experimental study. J. Chem. Phys. 2013, 138, 104310.

(9) Fano, U.; Macek, J. H. Impact Excitation and Polarization of the Emitted Light. Rev. Mod. Phys. 1973, 45, 553.

(10) Case, D. E.; Herschbach, D. R. Statistical theory of angular momentum polarization in chemical reactions. Mol. Phys. 1975, 30, 1537.

(11) Wade, E. A.; Lorenz, K. T.; Chandler, D. W.; Barr, J. W.; Barnes, G. L.; Cline, J. I. Ion imaging studies of product rotational alignment in collisions of $\mathrm{NO}\left(\mathrm{X}^{2} \Pi_{1 / 2}, j=0.5\right)$ with Ar. Chem. Phys. 2004, 301, 261-272.

(12) Cline, J. I.; Lorenz, K. T.; Wade, E. A.; Barr, J. W.; Chandler, D. W. Ion imaging measurement of collision-induced rotational alignment in Ar-NO scattering. J. Chem. Phys. 2001, 115, 6277-6280.

(13) Wen, B.; Meyer, H.; Kłos, J.; Alexander, M. H. Joint Experimental-Theoretical Investigation of the Lower Bound States of the $\mathrm{NO}\left(\mathrm{X}^{2} \Pi\right)-\mathrm{Kr}$ Complex. J. Phys. Chem. A 2009, 113, 7366-7375.

(14) Alexander, M. H. A new, fully ab initio investigation of the $\mathrm{NO}\left(\mathrm{X}^{2} \Pi\right)$ Ar system. I. 
Potential energy surfaces and inelastic scattering. J. Chem. Phys. 1999, 111, 74267434.

(15) Kłos, J.; Aoiz, F. J.; Menéndez, M.; Brouard, M.; Chadwick, H.; Eyles, C. J. Ab Initio studies of the interaction potential for the $\mathrm{Xe}-\mathrm{NO}\left(X^{2} \Pi\right)$ van der Waals complex: Bound states and fully quantum and quasi-classical scattering. J. Chem. Phys. 2012, 13\%, 014312.

(16) Bernstein, R. Atom-molecule collision theory: a guide for the experimentalist; Plenum Press, New York, USA, 1979.

(17) Bowman, J. M. Rotational rainbows in inelastic atom-molecule differential crosssections. Chem. Phys. Lett. 1979, 62, 309-311.

(18) Schinke, R.; Korsch, H. J.; Poppe, D. Rainbows in rotationally inelastic scattering: A comparative study of different model potential surfaces and dynamical approximations. J. Chem. Phys. 1982, 77, 6005-6020.

(19) Levine, R. D. Molecular Reaction Dynamics; Cambridge University Press, Cambridge, UK, 2005.

(20) Pirani, F.; Brizi, S.; Roncaratti, L. F.; Casavecchia, P. Beyond the Lenddard-JOnes model: a simple and accurate potential function probed by high resolution scattering data useful for moleular dynamics simulations. Phys. Chem. Chem. Phys. 2008, 10, 5489.

(21) Alexander, M. H. Rotationally inelastic collisions between a diatomic molecule in a ${ }^{2} \Pi$ electronic state and a structureless target. J. Chem. Phys. 1982, 76, 5974.

(22) Chandler, D. W.; Stolte, S. Chapter 5, Tutorials in Molecular Reaction Dynamics, Eds., M. Brouard and C. Vallance; Royal Society of Chemistry, Cambridge, UK, 2010. 
(23) Eppink, A. T. J. B.; Parker, D. H. Velocity map imaging of ions and electrons using electrostatic lenses: application in photoelectron and photofragment ion imaging of molecular oxygen. Rev. Sci. Instrum. 1997, 68, 3477-3483.

(24) Chandler, D. W.; Houston, P. L. Two-dimensional imaging of state-selected photodissociation products detected by multiphoton ionization. J. Chem. Phys. 1987, 87, 14451447.

(25) Aoiz, F. J.; Verdasco, J. E.; Herrero, V. J.; Rábanos, V. S.; Alexander, M. H. Attractive and repulsive interactions in the inelastic scattering of NO by Ar: A comparison between classical trajectory and close-coupling quantum mechanical results. J. Chem. Phys. 2003, 119, 5860 .

(26) HIBRIDON is a package of programs for the time-independent quantum treatment of inelastic collisions and photodissociation written by M. H. Alexander, D. E. Manolopoulos, H.-J. Werner, B. Follmeg, P. J. Dagdigian, and others. More information and/or a copy of the code can be obtained from the website http://www2.chem.umd.edu/groups/alexander/hibridon/hib43.

(27) Alexander, M. H. Differential and Integral Cross Sections for the Inelastic Scattering of NO $\left(\mathrm{X}^{2} \Pi\right)$ by Ar Based on a New Ab Initio Potential Energy Surface. J. Chem. Phys. 1993, 99, 7725-7738.

(28) Chapman, S.; Green, S. Rotational excitation of linear molecules by collisions with atoms: Comparison of classical and quantum methods. J. Chem. Phys. 1977, 67, 2317 (15 pages).

(29) Brouard, M.; Chadwick, H.; Gordon, S. D. S.; Hornung, B.; Nichols, B.; Kłos, J.; Aoiz, F. J.; Stolte, S. Fully quantum state resolved inelastic scattering of $\mathrm{NO}(\mathrm{X})+$ Kr: differential cross sections and product rotational alignment. J. Chem. Phys. 2014, submitted. 
(30) Alexander, M. H.; Dagdigian, P. J.; DePristo, A. E. Quantum Interpretation of Fully State-Selected Rotationally Inelastic Collision Experiments. J. Chem. Phys. 1977, 66, 59-66.

(31) de Miranda, M. P.; Aoiz, F. J.; Bañares, L.; Sáez-Rábanos, V. A unified quantal and classical description of the stereodynamics of elementary chemical reactions: Stateresolved $\boldsymbol{k}-\boldsymbol{k}^{\prime}-\boldsymbol{j}^{\prime}$ vector correlation for the $\mathrm{H}+\mathrm{D}_{2}(v=0, j=0)$ reaction. J. Chem. Phys. 1999, 111, 5368.

(32) de Miranda, M. P.; Aoiz, F. J.; Brouard, M.; Saez-Rabanos, V. Spatial distributions of angular momenta in quantum and quasiclassical stereodynamics. J. Chem. Phys. 2004, 121, 9830 .

(33) Brouard, M.; Hornung, B.; Aoiz, F. J. Origin of Collision-Induced Molecular Orientation. Phys. Rev. Lett. 2013, 111, 183202. 